\title{
RUEDAS VINCULANTES COMO ESPACIO DE INCLUSION EN EL CONTEXTO UNIVERSITARIO: LA EXPERIENCIA DE LA UNIVERSIDAD ESTATAL AMAZÓNICA, PUYO, PASTAZA, ECUADOR
}

\author{
Rosaura Gutiérrez Valerio de MAY* \\ Elisa LÓPEZ ${ }^{*}$ \\ Eluzinete PEREIRA** \\ Eduardo CAMPAÑA ${ }^{* * *}$ \\ Sandra SORIA ${ }^{* * * * *}$ \\ Thomas MAY*****
}

RESUMEN: En la Universidad Estatal Amazónica (UEA), en la Amazonía Ecuatoriana, se inició un programa de Desarrollo Humano Integral, aplicando la metodología de la TCI. Como primeros resultados de las Ruedas Vinculantes (término utilizado en el contexto universitario para la TCI), se presentan las apreciaciones de los participantes, que

Coordinadora Programa de Desarrollo Humano Integral. UEA - Universidad Estatal Amazónica. Via Napo km 21/2 Paso Lateral, Puyo - Ecuador. 032-888-118 rgutierrez@uea.edu.ec, rosauragmay@gmail.com

** Directora del departamento de Vinculación. UEA. Universidad Estatal Amazónica. Via Napo km 21/2 Paso Lateral, Puyo - Ecuador. 032-888-118 - elopez@uea.edu.ec

*** Formadora en terapia comunitaria. Muyumpa - Centro de Formación en Terapia Comunitaria Integrativa (Polo de la Mitad del Mundo). Quito - Ecuador. lusouza60@ yahoo.com

**** Formador en Terapia Comunitaria. Muyumpa - Centro de Formación en Terapia Comunitaria Integrativa (Polo de la Mitad del Mundo). Quito - Ecuador. educame1942@ yahoo.com

***** UEA - Universidad Estatal Amazónica. Via Napo km 21/2 Paso Lateral, Puyo Ecuador.032-888-118-rsoria@uea.edu.ec

****** Experto Integrado del CIM. UEA - Universidad Estatal Amazónica. Via Napo km 21/2 Paso Lateral, Puyo - Ecuador. 032-888-118 - may_gutierreztr@yahoo.es, tmay@uea.edu.ec 
resaltan que los temas tratados les sirven como aporte para mejorar sus propios problemas, que sienten alivio y mayor autoconfianza, así como mayor confianza y solidaridad con los compañeros de curso. Esto confirma que las sesiones contribuyen a la creación de redes solidarias.

PALABRAS CLAVE: Inclusión/redes solidarias. Interculturalidad. Educación superior.

\section{Introducción}

La Amazonía ecuatoriana es una región de altos niveles de pobreza, conflictos socioculturales y también socio-ambientales, y bajo grado de formación académica (ARIAS GUTIÉRREZ et al., 2015). Hay presencia de diez pueblos originarios (Achuar, Andoa, Cofán, Kichwa, Sápara, Sequoia, Shuar, Shiwiar, Siona y Waorani) que preservan sus culturas y la mayoría de ellos también sus lenguas. Existe una historia secular de exclusión de estos pueblos, primero por parte del poder colonial y después en la sociedad del estado ecuatoriano independiente, con su centro político, económico y cultural en Quito, ubicado en la región de la Sierra donde están radicadas las élites mestizas, dentro de un contexto físico, social y cultural bien distinto de la Amazonía.

Como señalan Arias Gutiérrez et al. (2015), el sector educativo, específicamente en su nivel superior, pero en principio en todos los niveles, tiene el potencial de contribuir a revertir esta situación de exclusión cultural, económica, lingüística y social, a través de la facilitación de conocimientos, capacidades y habilidades. De todos modos, en el mismo sector de educación superior existen determinadas barreras de exclusión, tanto de admisión como también después de haber conseguido matricularse en una carrera universitaria, que los jóvenes de las nacionalidades originarias amazónicas pueden superar, pero con dificultades (GUTIÉRREZ et al., 2015). En esta situación de exclusión, causada estructuralmente y en contradicción con los derechos garantizados en la constitución de 2008 (ECUADOR, 2014), el respeto y aprecio que se les da a las culturas y los conocimientos locales adquiere especial importancia, así como la capacidad de los jóvenes de formar redes solidarias de apoyo y ayuda mutua.

La Universidad Estatal Amazónica (UEA), con sede en Puyo, Provincia de Pastaza, fue establecida en 2002 como primer centro de educación superior en esta región. El segundo Plan Nacional para el Buen Vivir (2013-2017) (ECUADOR, 2013) establece en su objetivo 5, como 
prioridad del Ecuador "Construir espacios de encuentro común y fortalecer la identidad nacional, las identidades diversas, la plurinacionalidad y la interculturalidad." Dentro de este objetivo general, como objetivo específico 3, se expresa la necesidad de "Garantizar a la población el ejercicio del derecho a la comunicación libre, intercultural, incluyente, responsable, diversa y participativa." (ECUADOR, 2013).

La Universidad Estatal Amazónica (UEA) tiene como misión: "Generar ciencia, tecnología, formar profesionales y científicos, para satisfacer las necesidades de desarrollo sustentable, integral y equilibrado del ser humano, de la Región Amazónica y el Ecuador; conservando sus conocimientos ancestrales y fomentando su cultura." (UEA, 2015). Fundamentado por estos dos pilares, se propone la ejecución de un programa de Desarrollo Humano Integral en donde una de sus estrategias es contribuir con la generación de espacio de habla y de escucha en un contexto de respeto, diagnosticando las dificultades que se presentan a los estudiantes, pero igualmente promoviendo que el grupo genere sus propias alternativas de solución, tal como señala Camarotti (2014), de lo individual a lo grupal. Esta propuesta fue aceptada por el consejo universitario, permitiendo la realización en primer momento con los docentes para dar a conocer la metodología.

En ese contexto, con el apoyo del Polo Muyumpa, que es un centro de formación en Terapia Comunitaria Integrativa con sede en Quito, Ecuador, se comenzó en diciembre 2014 a desarrollar en esa universidad un programa de Terapia Comunitaria Integrativa, denominada "Ruedas Vinculantes", con irradiación hacia el sector de la educación secundaria. Los objetivos de este programa son de fomentar un espacio de habla y escucha entre docentes y estudiantes, y de facilitar la creación de redes solidarias de apoyo que permitan la búsqueda de soluciones en conjunto.

En el presente trabajo se relatan las primeras experiencias con estudiantes universitarios. El principal objetivo de la investigación cuyos primeros resultados se exponen aquí es de evaluar el potencial del método de la Terapia Comunitaria Integrativa para lograr los objetivos mencionados en el contexto universitario y en el contexto socio-cultural de la Amazonía ecuatoriana. En el presente trabajo se presentan los resultados obtenidos hasta finales de julio de 2015.

\section{Metodología}

La primera rueda se realizó en un curso de capacitación que se impartió por parte de la UEA a un público externo. Antes de iniciar el semestre 
de 2015 se realizó una rueda con los docentes de la UEA. Sin embargo, la gran mayoría de las ruedas se desenvolvieron con estudiantes de la carrera de Ingeniería Agropecuaria, en varios paralelos del primer y segundo curso, también en cursos superiores, y una rueda se realizó con un paralelo de nivelación que es un curso preparatorio, antes de insertarse los estudiantes en la carrera. Otra rueda se realizó con dos paralelos del primer curso de la carrera de Ingeniería en Turismo. La prioridad que se les dio a los cursos iniciales de las carreras tiene dos propósitos: En primer lugar, la fase inicial de la educación superior es un período crítico, en el que suelen surgir situaciones problemáticas crisis personales en los estudiantes, y cuando ellos toman decisiones que tiene consecuencias para su vida académica y extra-académica posterior, siendo esa fase un período crítico en la biografía (JOHNSTON, 2013). Por el otro lado, de esa forma iba a quedar abierta la posibilidad de realizar estudios longitudinales, a través del tiempo, para detectar posibles impactos positivos de las ruedas vinculantes en plazos de varios semestres o años.

En principio se había contemplado un plan piloto, aplicando un pre-test antes de iniciar las actividades de las ruedas vinculantes, como línea de base, y un post-test, después de haber concluido esas actividades. Se iba a iniciar el trabajo con las ruedas vinculantes solo en la carrera de Ingeniería Agropecuaria con dos cursos paralelos de primer semestre con un total de 60 estudiantes. La investigadora principal era la docente de la materia Lenguaje y Comunicación. Dos cursos paralelos con igual número de estudiantes no iban a participar en las ruedas vinculantes, para servir como grupo control. Sin embargo, debido a los resultados de apertura y detección de necesidades que iban presentando los grupos ya después de las primeras sesiones, se decidió con el director y otra docente de la carrera (Sandra Soria, co-autora de ese trabajo) ampliar las ruedas vinculantes a todos los semestres de la carrera de Ingeniería Agropoecuaria. Con esa decisión se alteró el diseño inicial cuasi-experimental del estudio.

A partir de las primeras experiencias se solicitó a las personas responsables del curso de nivelación universitaria y de la carrera de Turismo realizar una rueda con grupos en varias situaciones. Para esto, se acordó con los docentes de utilizar una hora del horario de sus unidades de clase. Para le ejecución de las ruedas, se siguió el patrón de la TCI que contempla: 1- Acogida y dentro de ella, bienvenida, que es la Terapia Comunitaria Integrativa (la rueda vinculante), celebraciones, reglas, dinámica de integración, 2- Selección del tema, por qué hablar, de que hablar, exposición del tema, identificación, votación y agradecimiento. 3-Contextualización. 
4- Problematización a partir de la pregunta mote (comodín o simbólico). 5- Ritual de cierre.

Luego del cierre se les pidió a los participantes de anotar las experiencias que tuvieron en las ruedas. A pesar de haber realizado más ruedas, solo se contemplan para el presente trabajo las ruedas de que al final se obtuvieron las apreciaciones de los y las participantes presentes, pues son las bases para el análisis. Una vez digitadas todas las apreciaciones, se agruparon las propuestas en categorías, según su contenido, entrando en ese paso elementos de interpretación de texto, que se enmarcan en el paradigma de investigación social cualitativa-interpretativa (ESPITIA, 2000; GONZÁLEZ MONTEAGUDO, 2001). Posteriormente se establecieron los porcentajes de los participantes cuyas respuestas correspondían a las diferentes categorías. Se agruparon las respuestas por género, y se realizó un test de chi cuadrado 2 × 2 para examinar si hay diferencias de género.

\section{Resultados}

\section{Temas propuestos y tratados}

En total, entre enero y julio 2015 en la Universidad Estatal Amazónica se realizaron 20 ruedas vinculantes con estudiantes universitarios, y una rueda con docentes del curso de nivelación. En la mayoría de las ruedas se propusieron cuatro o cinco temas, si bien en algunos casos solamente se propuso un tema o dos, y en un caso el número de temas propuestos llegó hasta a siete. El número total de temas propuestos en todas las ruedas fue de 85 .

Tabla 1 - Categorías de temas y sus frecuencias

\begin{tabular}{|l|c|c|c|c|}
\hline \multicolumn{1}{|c|}{$\begin{array}{c}\text { Categoría } \\
\text { de temas }\end{array}$} & $\begin{array}{c}\text { Número de veces } \\
\text { que un tema } \\
\text { fue propuesto }\end{array}$ & $\%$ & $\begin{array}{c}\text { Número de veces } \\
\text { que un tema } \\
\text { fue elegido }\end{array}$ & $\%$ \\
\hline $\begin{array}{l}\text { Sufrimiento } \\
\text { psíquico }\end{array}$ & 20 & 23,5 & 6 & 24,0 \\
\hline $\begin{array}{l}\text { Conflictos } \\
\text { interpersonales }\end{array}$ & 20 & 23,5 & 4 & 16,0 \\
\hline Estrés & 16 & 18,8 & 3 & 12,0 \\
\hline $\begin{array}{l}\text { Problemas } \\
\text { personales }\end{array}$ & 14 & 16,5 & 5 & 20,0 \\
\hline
\end{tabular}




\begin{tabular}{|l|c|c|c|c|}
\hline \multicolumn{1}{|c|}{$\begin{array}{c}\text { Categoría } \\
\text { de temas }\end{array}$} & $\begin{array}{c}\text { Número de veces } \\
\text { que un tema } \\
\text { fue propuesto }\end{array}$ & $\%$ & $\begin{array}{c}\text { Número de veces } \\
\text { que un tema } \\
\text { fue elegido }\end{array}$ & $\%$ \\
\hline $\begin{array}{l}\text { Relaciones } \\
\text { intra-grupales }\end{array}$ & 9 & 10,6 & 3 & 12,0 \\
\hline $\begin{array}{l}\text { Problemáticas } \\
\text { sociales } \\
\text { más generales }\end{array}$ & 6 & 7,1 & 4 & 16,0 \\
\hline
\end{tabular}

Fuente: Elaboración propia.

20 de los temas propuestos (23.5\%) se categorizaron como sufrimiento psíquico. Entre ellos predominaban temas relacionados con sentimientos de separación de la familia (15). Igualmente, 20 temas $(23.5 \%)$ se categorizaron como relacionados con conflictos interpersonales, en su mayoría en la familia (15), pero también en la pareja (5). 16 temas $(18,8 \%)$ se atribuyeron a aspectos relacionados con el estrés, y entre ellos predominaban aspectos del estrés causado por diferentes exigencias poco compatibles (9), antes del estrés causado por los estudios (6), que en varios casos se relacionaban con determinadas materias, específicamente física y matemática.

14 temas $(16,5 \%)$ se categorizaron como problemas personales del proponente relacionados con afecciones de la salud, temor de caer en problemas de alcohol y drogas, deudas económicas, distracciones en clase por el uso de medios de comunicación, timidez de hablar delante del grupo, dificultades relacionadas con metas y valores. Nueve temas $(10,6 \%)$ se categorizaron como vinculados a las relaciones dentro de los grupos (falta de compañerismo, conflictos intra-gupales, hablar atrás de la espalda de otros, etc.). En este contexto hay que mencionar que en la rueda con docentes del curso de nivelación, los cuatro temas se referían a temas de esa categoría. Seis temas $(7,1 \%)$ se categorizaron como temas de problemática social con un fondo más general, como feminicidio, violencia, deterioro del medio ambiente.

En cuanto a los temas que fueron priorizados por los participantes, también fueron más votados aspectos relacionados con sufrimiento psíquico ( 6 ruedas, equivalente a $24,0 \%$ ). La segunda categoría más priorizada fue la de problemas personales ( 5 ruedas, 20,0\%), seguido de temas relacionados con estrés (3 ruedas, 12,0\%) y problemas grupales ( 3 ruedas, $12,0 \%)$. Temas relacionados con conflictos familiares o de pareja, que for- 
man parte de la categoría de los conflictos interpersonales, fueron priorizados en dos de las 25 ruedas $(8,0 \%)$.

\section{Apreciaciones por los y las participantes}

En las 14 ruedas que fueron realizados durante el periodo marzo/julio 2015 , con un total de 272 participantes, de los cuales 161 correspondían al sexo femenino y 111 al sexo masculino, fue realizada una encuesta para examinar las apreciaciones por los participantes. Sin excepción, las experiencias fueron valoradas como positivas por los y las participantes. $42.3 \%$ seńaló que en las ruedas se aportan soluciones, ayudan a mejorar. $39.4 \%$ dijo que las ruedas les permite expresar problemas, sentir alivio, levantar el ánimo, tener confianza en uno mismo. Un $29.4 \%$ de los y las participantes señalaron que las ruedas permiten generar más confianza y solidaridad con los compañeros, y el $22.7 \%$ expresó que les permite conocer mejor los compañeros. $15.6 \%$ expuso que no es fácil tener suficiente confianza para compartir su problemática personal. Un 33\% manifestó como es bueno que se vuelvan a repetir las ruedas (tabla1).

En los tres aspectos que fueron expresados con mayor frecuencia - las ruedas aportan soluciones que ayudan a mejorar, se siente alivio y se permite a expresar problemas, y se mejora la solidaridad con los compañeros - se observa que el porcentaje de las participantes del sexo femenino que se expresaron en este sentido fue más alto que el porcentaje de los participantes masculinos. Aunque la tendencia es clara, la diferencia entre ambos sexos en ningún caso fue significativa, según la prueba de chi cuadrado en una tabla de $2 \times 2$.

Tabla 2 - Categorías de valoración de la experiencia

\begin{tabular}{|l|c|c|c|}
\hline & mujeres & hombres & total \\
\hline \multirow{2}{*}{ Se aportan soluciones, me ayudan a mejorar } & 77 & 39 & 116 \\
\cline { 2 - 4 } & $47,8 \%$ & $34,8 \%$ & $42,6 \%$ \\
\hline $\begin{array}{l}\text { Expresar problemas, sentir alivio, levantar el } \\
\text { ánimo, tener más confianza en uno mismo }\end{array}$ & 70 & 38 & 108 \\
\cline { 2 - 4 } & $43,5 \%$ & $34,2 \%$ & 39,4 \\
\hline $\begin{array}{l}\text { Más confianza y solidaridad con los } \\
\text { compañeros }\end{array}$ & 52 & 28 & 80 \\
\hline & $32.3 \%$ & $23,7 \%$ & $29,4 \%$ \\
\hline
\end{tabular}


Eduardo Campaña, Sandra Soria e Thomas May

\begin{tabular}{|l|c|c|c|}
\hline & mujeres & hombres & total \\
\hline \multirow{2}{*}{ Conocer mejor a los/las compañeros/as } & 20 & 23 & 43 \\
\cline { 2 - 4 } & $22,4 \%$ & $23,4 \%$ & $22.7 \%$ \\
\hline \multirow{2}{*}{ No es fácil tener suficiente confianza } & 24 & 16 & 40 \\
\cline { 2 - 4 } & $15,9 \%$ & $15,1 \%$ & $15,6 \%$ \\
\hline \multirow{2}{*}{ Que se vuelvan a repetir las ruedas } & 19 & 14 & 33 \\
\cline { 2 - 4 } & $11,8 \%$ & $12,6 \%$ & $12.1 \%$ \\
\hline
\end{tabular}

Fuente: Elaboración propia.

\section{Discusión}

En los temas, tanto en los que se propusieron como en los que los participantes finalmente eligieron para tratar en la rueda, destaca la predominancia del sufrimiento psíquico. El estrés se propuso y se eligió de forma relativamente menos frecuente, como también los problemas intra-grupales de los cursos Esto se puede interpretar de forma que los sufrimientos psíquicos suelen ser los problemas más acuciantes para los estudiantes, más que el estrés causado por los estudios y por otros motivos, y más por los problemas de dinámica de grupo en los cursos.

A pesar de que la cultura amazónica y andina tiene la fama de poco usar la comunicación abierta y directa, en el contexto de las ruedas no hubo mayores dificultades para lograr que los jóvenes comenzaran a hablar de sus problemas personales, y a ver que otros tienen los mismos problemas. Este último aspecto fue valorado por los participantes como una experiencia muy buena, que les ayudó a conocer más sus compañeros, y a sentir más confianza y solidaridad con ellos.

La gran valoración por los participantes de que "se reciben buenos consejos", podría parecer contradictoria a los principios de la TCI, ya que una de sus reglas justamente es de no dar consejos. En realidad, en todas las ruedas se cuidó y se respetó este principio. Las apreciaciones demuestran que los comentarios sobre experiencias propias, que surgen en la fase de la problematización de las sesiones de ruedas, fueron acogidas por otros participantes como aportes para trabajar sus propias problemáticas. De esa manera fueron percibidos como consejos que les ayudaban, a darse cuenta que no sólo ellos o ellas pasaban por semejante situación, lo que se traducía en un gran alivio. Frases como "Y, pensar que solo yo creía tener esa situación", fueron muy comunes escucharlas después cuando la terapeuta se encontraba con algunos de los participan- 
tes en algún pasillo o corredor. En la Terapia Comunitaria Integrativa, varios de sus pilares basados en la teoría sistémica, resiliencia y antropología cultural sostienen que donde está el problema está la solución, pues quien ya ha vivido una situación que se relaciona con el tema de la rueda y que ha superado, dentro de ese espacio que se genera para compartir a través de la pregunta mote, muchas veces se anima a socializarla. De esa manera surgen las problemáticas traducidas en perlas, lo que indudablemente permite generar un vínculo de solidaridad y apoyo, el cual se fortalece en el cierre con el "me balanceo pero no me caigo, pues mi grupo está ahî” (BARRETO, 2015).

Las apreciaciones realizadas en el campus universitario, la demanda que va teniendo de otros docentes y de los mismos estudiantes - en algunos de los casos solicitan formarse como terapeutas - permiten deducir que las ruedas vinculantes efectivamente pueden ser un medio para el manejo situaciones estresantes y de facilitar espacios de socialización, conexión y vínculos para redes solidarias en el contexto universitario. Además se pone de manifiesto que el alivio que los participantes experimentaron al hablar de sus problemas resulta en una mayor autoconfianza. Esto permite formular la hipótesis que las ruedas vinculantes tienen un potencial para generar impactos positivos, no solamente en el bienestar estudiantil y en actitudes solidarias, sino también para mejorar el rendimiento académico, al tener la mente menos ocupada por las preocupaciones, tal como comunicaron varios de los participantes. Se puede esperar que la integración de las ruedas en la práctica de enseñanza-aprendizaje de todas las carreras tenga impactos positivos en el éxito académico dentro del sistema de educación universitario.

La diversidad de culturas y lenguas - hay estudiantes de nacionalidades indígenas cuya lengua materna no es el castellano - , las diferentes regiones de donde llegan los y las estudiantes, genera muchas veces un clima que amerita de herramientas pertinentes para el abordaje de situaciones generadoras de sufrimiento. Existen ejemplos promisorios de escuelas de Brasil, donde en el sector de educación primaria se estableció la Terapia Comunitaria Integrativa en el currículum escolar, y constituye un espacio de diálogo, democrático e igualitario, que sirve para la estructuración de la planificación pedagógica (REIS; SALERNO 2011). Hace falta la aplicación y la validación de algunos instrumentos que puedan ser aplicados antes, en medio y al final del acompańamiento de las ruedas vinculantes para determinar hasta qué punto se sostienen y amplifican estos indicadores de una dinámica positiva que durante esos meses han aflorado. 


\section{Conclusiones}

Los resultados obtenidos señalan que se puede esperar que las ruedas vinculantes sean un método muy valioso, que no solamente tiene un impacto positivo en la salud integral de los estudiantes, sino también ayuda a mejorar el rendimiento académico y reducir la cuota de abandono universitario, fomentando la inclusión. Además puede servir como herramienta para diagnosticar las problemáticas que más apresuran a los estudiantes, con la ventaja que ofrece una transparencia a los participantes y genera vínculos solidarios entre ellos.

CHATTING CIRCLES AS A SPACE FOR INCLUSION IN THE UNIVERSITY CONTEXT: THE EXPERIENCE OF AMAZON STATE UNIVERSITY, PUYO, PASTAZA, ECUADOR

ABSTRACT: In the Amazon State University (UEA) in the Ecuadorian Amazon, a program was initiated Integral Human Development, applying the methodology of the TCI. As first results of the Chatting Circles (term used in the university context for TCI), the findings of the participants, highlighting that the topics serve them as a contribution to improve their own problems, they feel relief and greater confidence present, as well as greater confidence and solidarity with classmates. This confirms that the meetings contribute to the creation of solidarity networks.

KEYWORDS: Inclusion. Solidarity networks. Intercultural relationships. Higher education.

\section{BIBLIOGRAFÍA}

ARIAS GUTIÉRREZ, R. I. et al. Aprovechamiento de la agrobiodiversidad amazónica ecuatoriana y formación de capital humano. Revista Geográfica Venezolana, Venezuela, v.56, n.2, p.205-220, 2015.

BARRETO, A. Terapia comunitaria integrativa paso a paso. Ecuador: Edición para Ecuador, 2015.

CAMAROTTI, M. H. Terapia comunitaria integrativa: de lo individual para lo grupal - es el desafío. Quito: Muyumpa, Centro de Formación en Terapia Comunitaria Integrativa, 2014. (Curso de formación). 
ECUADOR. Constitución. Disponible en: <http://educacion.gob.ec/ wp-content/uploads/downloads/2012/08/Constitucion.pdf>. Acceso en: Dic. 2014.

ECUADOR. Secretaría Nacional de Planificacíon y Desarrollo. El segundo Plan Nacional para el Buen Vivir (2013-2017). Quito, 2013. Disponible en: <http://documentos.senplades.gob.ec/Plan $\% 20$ Nacional\%20Buen\%20Vivir\%202013-2017.pdf>. Acceso en: Jun. 2015.

ESPITIA, E. C. La fenomenología interpretativa como alternativa apropiada para estudiar los fenómenos humanos. Investigación y educación en enfermería, Medellín, v.18, p.27-35, 2000.

GONZÁLEZ MONTEAGUDO, J. El paradigma interpretativo en la investigación social y educativa: nuevas respuestas para viejos interrogantes. Cuestiones Pedagógicas, Sevilla, v.15, p.227-246, 2001.

GUTIÉRREZ, R. et al. Higher education and indigenous nationalities: challenges for inclusion in the Ecuadorian Amazonian region. Creative Education, Irvine, v.6, p.847-854, 2015.

JOHNSTON, B. El primer ańo de universidad: una experiencia positiva de transición. Madrid: Narcea, 2013.

REIS, M. L. A.; SALERNO, G. Educação escolar e terapia comunitaria integrativa: uma relação possível mediada pela pedagogía de Paulo Freire. In: CAMARORTI, M. E.; FREIRE, T. C. G. P.; BARRETO, A. P. (Ed.). Terapia comunitária integrativa sem fronteiras: compreendendo suas interfaces e aplicações. Brasília: MISMEC-DF, 2011. p.363 - 381.

UNIVERSIDAD ESTATAL AMAZÓNICA [UEA]. Misión. Disponible en: <http://www.uea.edu.ec/index.php/inicio/2013-09-24-08 -36-52/2013-09-24-08-39-46>. HYPERLINK "http://www.uea.edu.ed/ index.php/inicio/2013-09-24-08-36-52/2013-09-24-08-39-46.\%20 Consultado\%20Junio\%202015” Acceso en: jun. 2015. 
